\title{
Material Properties of Wire for the Fabrication of Knotted Fences
}

\author{
Dirk J. Pons, Gareth Bayley, Christopher Tyree, Matthew Hunt, and Reuben Laurenson \\ Department of Mechanical Engineering, University of Canterbury, Private Bag 4800, Christchurch 8020, New Zealand \\ Correspondence should be addressed to Dirk J. Pons; dirk.pons@canterbury.ac.nz
}

Received 5 May 2014; Accepted 29 October 2014; Published 26 November 2014

Academic Editor: Francisca Caballero

Copyright (C) 2014 Dirk J. Pons et al. This is an open access article distributed under the Creative Commons Attribution License, which permits unrestricted use, distribution, and reproduction in any medium, provided the original work is properly cited.

This paper describes the materials properties of galvanised fencing wire, as used in the fabrication of knotted wire fences. A range of physical properties are investigated: tensile strength, ductility in tension, Young's modulus, three-point bending, and bending span. A range of commercially available wire products were tested. The results show that most, but not all, high tensile wire samples met the minimum tensile and ductility requirements. Young's modulus results failed to provide any meaningful insights into wire quality. Flexural modulus results also failed to provide any insight into wire quality issues, with no statistically significant differences existing between acceptable and problematic wire batches. The implications are that premature fence failures are unlikely to be caused solely by reduced tensile properties. Existing test methods, including tensile strength and ductility, are somewhat incomplete, perhaps even unreliable, as measures of wire quality.

\section{Introduction}

Knotted wire fencing, despite being agriculturally ubiquitous, has a limited research literature. There are several aspects to the problem, including the manufacturing of the fencing, its erection, and its in-field use. The focus of the present paper is on the manufacturing, and the particular area under examination is the physical properties of wire.

\section{Background}

Knotted wire fencing, which is differentiated from single strands, diamond (chain link), welded mesh, and hexagonal mesh (chicken mesh), is a rectangular knotted mesh that is used for livestock retention, for example, sheep and deer. The fencing is an integral feature of the stock farming landscape worldwide and serves its purpose for years and even decades totally exposed to the environment. It is such a common product as to be overlooked. Yet the fabrication of this product is a sophisticated task that requires specialist machines [1-4]. Modern machines are numerically controlled to permit different configurations, for example, sizes of the rectangular openings. There are other challenges too: the steel wire used for fences tends to have high tensile strength, and this makes it particularly difficult to form the knotted features, since plastic deformation is required. Consequently high localized forces are required to bend the wire, and the wire needs to have the necessary material properties to accommodate this without fracture. Breakages of wire inside the fence knotting machine is highly problematic from a production perspective, since the high speed of operation causes wire to be jammed and tangled in the machine, and the high tensile strength of the material makes it difficult to extract. The machines also become damaged in these events. This results in the machine being out of production for an extended period, which adversely affects the production economics. Consequently the quality of the input wire is an important factor in the production process. The wire quality is also important in that it affects the fence erection processes. Similarly breakages of fencing in-field can have significant repercussions for the contractors who erect the fence or the agricultural operations of live-stock retention. This is an unsatisfactory customer experience. The fence manufacturer therefore wants to ensure that the raw wire stock is suitable for both fabrication and erection. Against this is the opposing pressure to reduce the cost of the input wire, which has negative effects on wire quality. The issue from the engineering perspective is therefore to obtain a wire feedstock that is fit for purpose: has sufficient material properties to withstand the fabrication and 
erection processes, without properties that are excessive (and therefore unnecessarily costly) to the process.

Standards do exist to control the quality of the input wire, and their foci are tensile strength and ductility; for example, see [5-9]. Corrosion resistance, hence also coating properties, is the other main strand of research [10-18] but is peripheral to the breakage problem considered here.

At present wire quality is only controlled by tensile strength and coating properties. However, these metrics are poor predictors of the performance of the wire in the manufacturing process in that wire that passes these tests can still fail in the machines [19]. The problem is that the production capability of the machines has increased such that wire failure is now a significant event, and the standards, which are mostly based on a previous generation of technology, are not providing the necessary quality control. Consequently there is a need to identify critical wire properties that can be used to differentiate between acceptable and poor wire quality, before it is input to the knotting machines.

\section{Purpose and Approach}

The two conventional materials properties used in the fencing industry are tensile strength and percent elongation (ductility) [6-9]. However the role of tensile strength and elongation, and their possible role in premature fence failures, is unclear. The objective of this particular paper was to apply a basket of physical-material tests to a set of wire samples of known history and infer the efficacy of the various tests in distinguishing wire quality. The background to the project is described in [19] along with a summary of some of the other tests.

The approach taken was first to obtain samples of manufactured fences and coils of input wire, of known acceptable and problematic product. These were commercially available products. The quality attributes of the wire were identified by the manufacturer (South Fence Machinery Ltd, New Zealand) and categorised as "acceptable" if it had not broken during fence fabrication or erection or "problematic" if it had failed in one or both situations. The quality categorisation thus supplied to the researchers at the outset was as follows: Acceptable: all Onesteel wire batches, Bekaert wire batches; Problematic: pacific wire breaking rolls 1, 2, and HiSPAN and Hurricane wire batches. To reduce external variability, all fence panels were produced by the same fence fabricator ("Producer") on machines from the manufacturer.

Samples were of complete fence panels and coils and covered a variety of input wire batches and brands. Assay pieces were cut from these fences or coils and subject to a variety of material tests. These were then statistically examined and compared to the fate of the fence/coil (where known), to determine the sensitivity of the test. Several tests were conducted, and the method for each is given preceding the results.

\section{Results}

4.1. Tensile Testing. Many materials properties can be calculated from undertaking tensile tests on wire specimens.

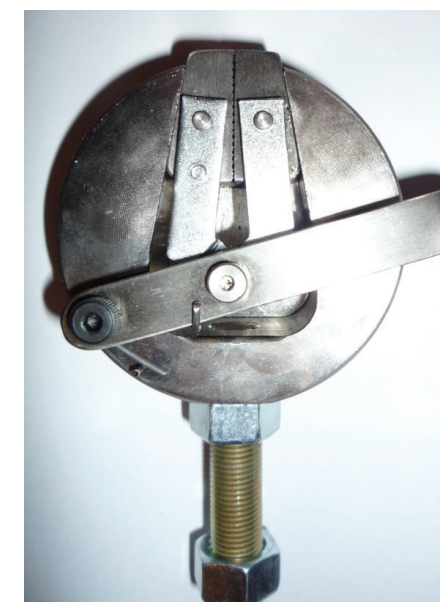

FIgure 1: Clamps for elongation test.

Firstly, the ultimate tensile strength (UTS) of wire can be determined by measuring the maximum force required to fracture the specimen in tension. Secondly, the addition of gauge marks before a tensile test allows the percentage elongation (measure of ductility) to be evaluated. The wire stiffness, measured by Young's modulus $(E)$, can also be inferred from the stress-strain curve. The tensile results may then be compared against the relevant wire standards, to assess compliance.

4.1.1. Experimental Method. Tensile tests were undertaken in accordance with the procedures set by ASTM A37005 [20]. Wire specimens to be tested were cut to $380 \mathrm{~mm}$ lengths. A gauge length of $250 \mathrm{~mm}$ was used to enable ductility calculations to be conducted. Special-purpose wire clamps were used to grip the wire without introducing stress concentrations, as shown in Figure 1. These clamps ensured that the final fracture position would occur inside the gauge length. However, since these clamps worked on a wedge tightening principle, the strain values (and hence Young's modulus) calculations were significantly affected. In some cases, separate tests were conducted using standard clamps to find Young's modulus. At least three samples per wire batch were tested, to gain a representative average for the batch.

Tensile tests were also conducted on line wires cut from fabricated fence samples. For these samples, the knots and stay wires were cut off, and a sample containing two crimps and one knot was tested. Due to the presence of the crimps, ductility measurement was not possible.

The wire specimens were mounted in a MTS 810 Materials Testing System. The rate of cross-head displacement was set at $6 \mathrm{~mm} /$ minute until failure. After failure two parts of the specimen were matched back together and the new gauge length was measured to obtain $\Delta L$, the change in gauge length after fracture. The elongation of the specimen is then $\Delta L / L$ where original specimen length $L=250 \mathrm{~mm}$ in this case. The ultimate tensile strength (UTS) was calculated as the maximum tensile force relative to the average wire diameter before testing. Young's modulus was calculated from the slope of the elastic region of the stress-strain curve. 
TABLE 1: Statistical high tensile UTS results for ANOVA: single factor test.

\begin{tabular}{lccccc}
\hline Source of variation & SS & df & MS & $F$ & $P$ value \\
\hline Between groups & 709486.1 & 12 & 59123.8 & 18.661 & $2.193 E-15$ \\
Within groups & 177417.4 & 56 & 3168.2 & & 1.930 \\
\hline Total & 886903.6 & 68 & & & \\
\hline
\end{tabular}

From the multiple tests carried out for each supplier, averages were found and statistical analyses performed: box plots, single factor ANOVA tests, and multiple comparisons of means.

4.1.2. Statistical Method. The one-way ANOVA technique tests the null hypothesis that two or more groups are drawn from the same population. For this investigation the null hypothesis is that no difference in tensile properties exists across the different wire batches (all batches are from the same "population" of wire). To confirm/deny the null hypothesis, the ANOVA test produces an $F$ statistic (ratio of the variance calculated among the means to the variance within the samples). If the group means are drawn from the same population, then the central limit theorem infers that the variance between the group means should be lower than the variance of the samples. Therefore, a higher $F$ statistic (ratio) implies that the samples are from different populations, and thus the null hypothesis can be rejected. If $F>F_{\text {crit }}$, then the null hypothesis can be rejected at the $\alpha \%$ confidence level. ANOVA cannot be used to determine which groups are "statistically different." For this, a multiple comparison of means study was carried out, using confidence intervals for mean differences.

4.1.3. Wire Samples. From discussions with the producer, it was evident that no significant problems had been encountered with Onesteel wire, either during fabrication or in service. The majority of the wire quality issues had arisen around the use of certain Pacific Steel wire batches, namely, the Pacwire-HiSPAN, -breaking roll 1, and -breaking roll 2 samples. Later on in the year, a fencing contractor from Matawai (Gisborne, New Zealand) was experiencing wire breakages during tie off procedures when using Hurricane wire. Two different roll samples were obtained for testing purposes. In the accompanying figures and tables, the identified known acceptable wire is indicated in green whilst wire from failed fences is labelled problematic and indicated in red. Any other wire tested is for comparative purposes and is indicated in a neutral (blue) colour.

4.2. Results for Tensile Strength. The UTS results for each grade of wire are compiled below. Tests are named via the tag supplied with the wire coil. Tests performed on the fence after manufacture (i.e., wire cut from a fabricated fence) are named "after" and thus any other tests can be assumed to be on coiled wire.

4.2.1. UTS for High Tensile Wire. NZS 3471:1974 stipulates that $2.5 \mathrm{~mm}$ HT wire must have a minimum UTS of $1235 \mathrm{MPa}$ (or minimum breaking force of $6060 \mathrm{~N}$ ). As shown in

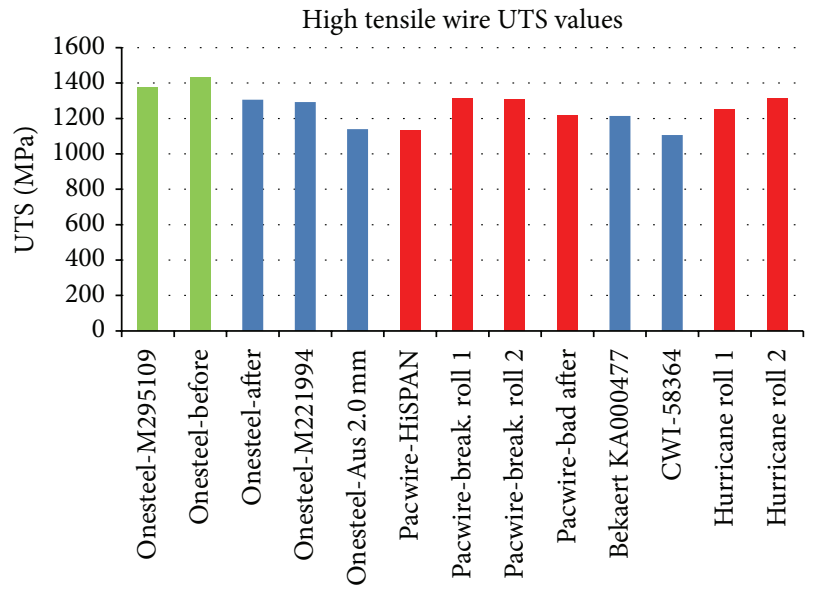

FIGURE 2: Average UTS results for a range of high tensile wire batches.

Figure 2, several wire batches exceeded this standard, which is good. The Onesteel "before" wire batch had the greatest UTS at $1433 \mathrm{MPa}$ (average over the 8 different batches tested). The Pacific Steel HiSPAN batch failed to meet the minimum standard, scoring an average UTS of $1131 \mathrm{MPa}$. The CWI batch registered the lowest UTS, with an average of $1106 \mathrm{MPa}$ being recorded. Interestingly, both of the problematic Hurricane wire rolls surpassed the minimum standard.

The accompanying boxplot in Figure 3 is used to show the variance of the wire and to look for statistical differences between batches. Of most importance are the results given by Pacific wires HiSPAN batch. Here the wire is observed to have a high variance as shown by the long box and whiskers. This shows that the UTS of this batch is highly variable and cannot be relied on to consistently meet the standard of $1235 \mathrm{MPa}$. Furthermore the upper range of UTS for HiSPAN wire is still below that of any Onesteel brand tested. This indicates a clear difference in tensile strength between the different product batches.

The statistical ANOVA analysis gives a statistical test of whether the means of several groups are all equal (the null hypothesis). For this investigation, a significance level of $5 \%$ $(\alpha=0.05)$ was chosen. Table 1 shows that the $F$ value for the test (18.661) is greater than $F_{\text {crit }}(1.930)$. This gives a strong indication that the UTS values from the different wire brands/batches are not the same (i.e., null hypothesis can be rejected). Since the ANOVA test cannot determine which particular pairs are "significantly different," a multiple comparison procedure was carried out, using the results from the ANOVA test. Figure 4 shows that there are 34 significantly different mean pairs, the largest being between Onesteel "before" and Pacific Steel HiSPAN batches. 


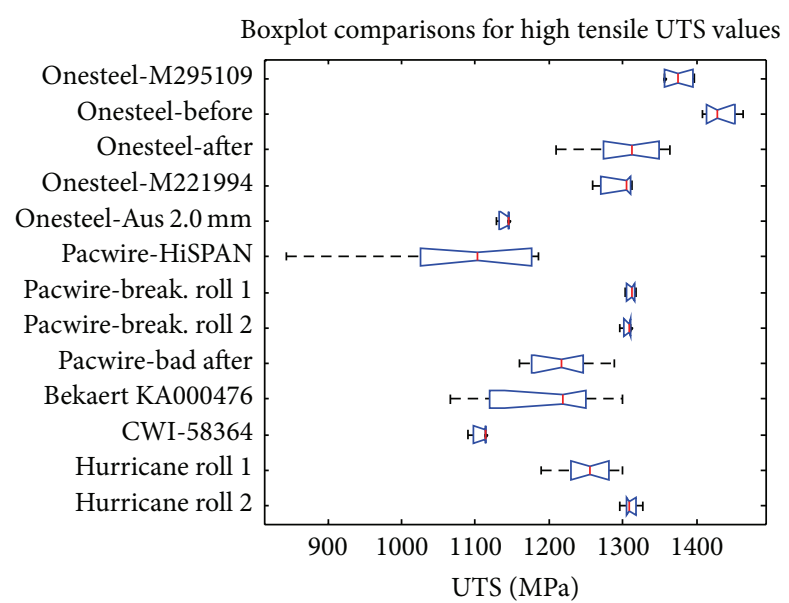

FIgURE 3: Boxplot comparison between the various high tensile wire UTS batches.

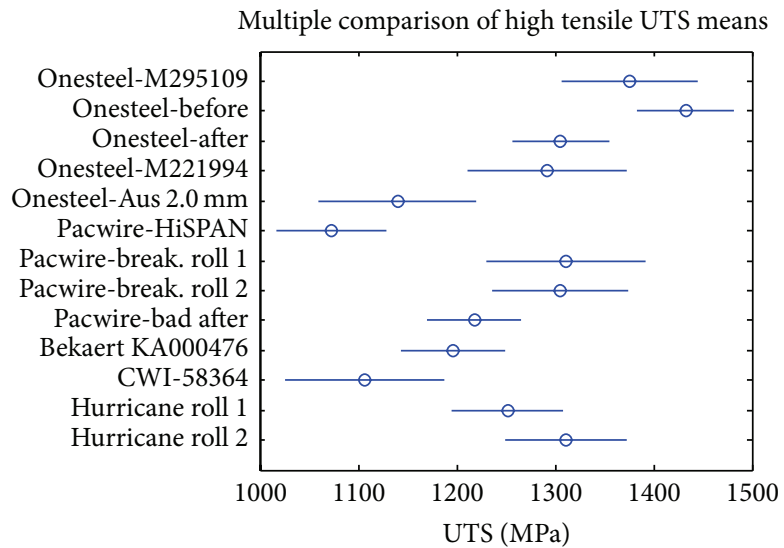

FIGURE 4: Multiple comparison of UTS means for high tensile wire batches.

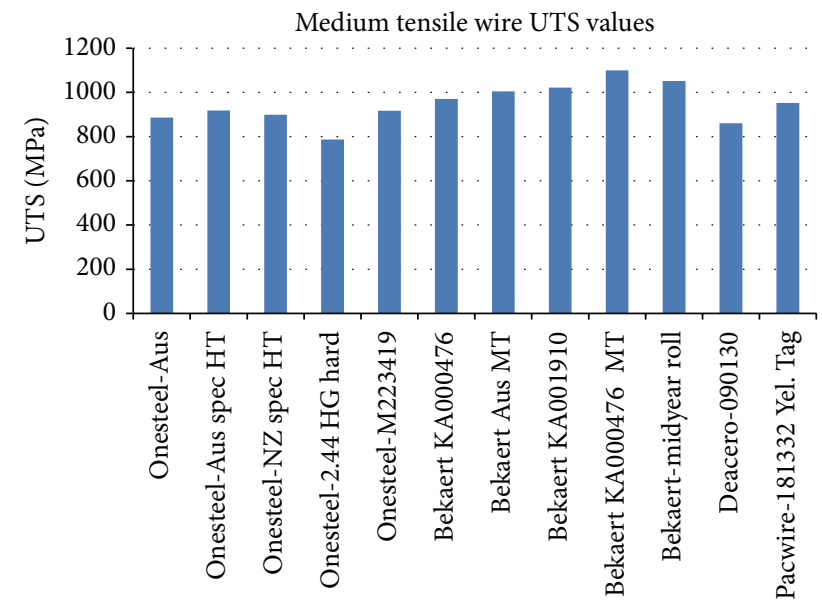

FIGURE 5: Average UTS results for a range of medium tensile wire batches.

4.2.2. UTS for Medium Tensile Wire. Figure 5 shows that the medium tensile wire all performed well over the minimum value of $600 \mathrm{MPa}$ for $2.5 \mathrm{~mm}$ diameter wire [21]. That standard also states the following: "Tensile strength range from any
1 batch within a type of wire shall not be more than 200 Mpa." From the boxplot in Figure 3, it is evident that only the Pacific steel HiSPAN batch failed to meet this criterion.

4.2.3. Soft Wire. NZS 3471:1974 stipulates that $2.5 \mathrm{~mm}$ mild steel (soft) wire should exceed UTS of $430 \mathrm{MPa}$. Figure 6 shows that this standard is exceeded by all batches, except for some $4.0 \mathrm{~mm}$ wire intended for producing staples that were included for interest.

4.3. Percent Elongation (Ductility). The percentage elongation (ductility) results for each grade of wire are assembled below. Tests are named via the tag supplied with the wire coil. Batches match those tested in the UTS tests.

4.3.1. Ductility of High Tensile Wire. Figure 7 shows the percent elongation (ductility) results from the high tensile wire batches tested. NZS 3471:1994 stipulates that the ductility (over a $250 \mathrm{~mm}$ gauge) should not fall below $4 \%$. A minimum amount of ductility is required to prevent brittle failures, but too much ductility makes the wire prone to stretching (resulting in baggy fences). Figure 6 shows that the problematic Pacific steel batches feature the lowest ductility. Indeed, 
TABLE 2: Statistical high tensile \% elongation results for ANOVA: single factor test.

\begin{tabular}{lccccc}
\hline Source of variation & SS & df & MS & $F$ & $P$ value \\
\hline Between groups & 46.3 & 10 & 4.631 & 41.394 & $1.811 E-15$ \\
Within groups & 3.58 & 32 & 0.112 & & 2.142 \\
\hline Total & 49.89 & 42 & & & \\
\hline
\end{tabular}

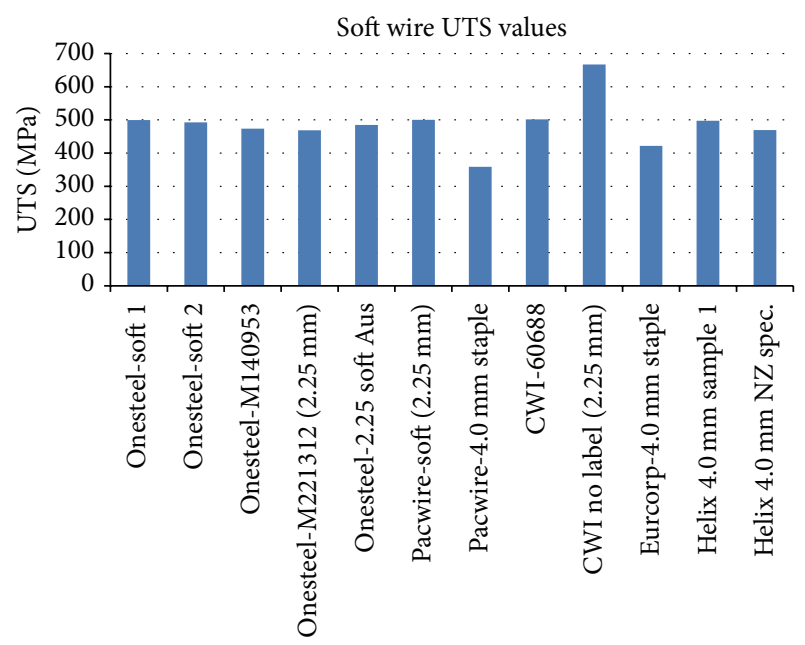

FIgURE 6: Average UTS results for a range of soft wire batches.

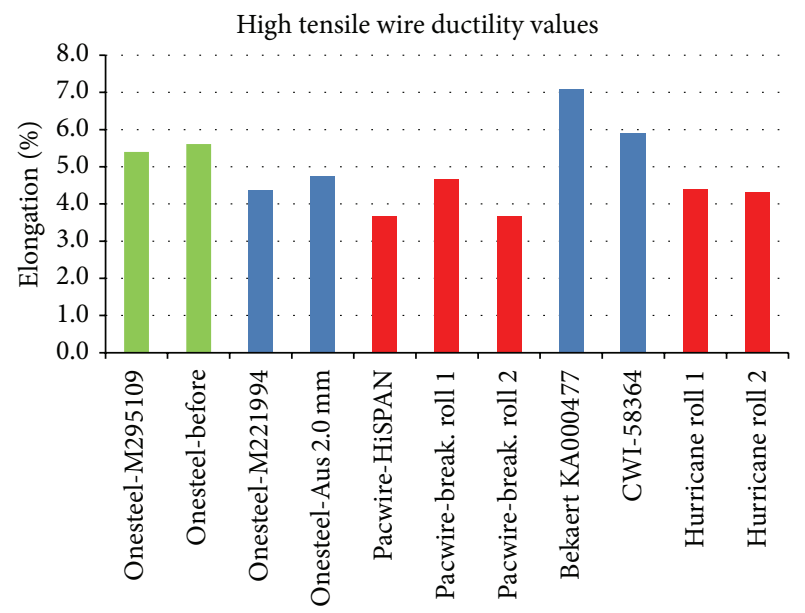

FIgURE 7: Average percent elongation results for a range of high tensile wire batches.

both the HiSPAN and breaking roll 2 batches fail to meet the $4 \%$ minimum ductility threshold. The acceptable Onesteel batches both have superior ductility, at approximately 5.5\%. Bekaert and CWI which had both performed averagely in the UTS test showed large amounts of elongation with $7 \%$ and $5.9 \%$, respectively. The results for Hurricane wire batches show that despite the user's complaint of "brittleness," both have a ductility of $4.35 \%$ which is above the required minimum.

The accompanying boxplot in Figure 8 shows that there is less variation between tests, as indicated by narrower whiskers. The highest variations were observed within the Onesteel "before" and Hurricane roll 2 wire batches. Table 2 shows that the $F$ value for the test (41.394) is greater than $F_{\text {crit }}(2.142)$. This gives a strong indication that the ductility values from the different wire brands/batches are not the same (i.e., null hypothesis can be rejected). Since the ANOVA test cannot determine which particular pairs are "significantly different," a multiple comparison procedure was carried out, using the results from the ANOVA test. Figure 9 shows that there are 29 significantly different mean pairs, the largest being between Bekaert KA000467 and Pacific steel HiSPAN/breaking roll 2 batches.

4.3.2. Ductility of Medium Tensile Wire. For the medium tensile wire shown in Figure 10, the majority of the brands were found to have around $6 \%$ ductility. However, two batches were found to fall below the $4 \%$ threshold. The first batch is the Bekaert KA001910 with 3.7\% ductility, while the worst is the Pacific steel 181332 yellow tag batch with 1.13\% ductility. In the case of the Bekaert batch, it has a relatively high UTS which could explain the reduction in ductility. However, the yellow tag Pacific steel batch is likely to suffer from microstructural defects (i.e., inclusions or grain size effects). The Deacero batch is interesting, displaying a $10.3 \%$ ductility. Its UTS is $860 \mathrm{MPa}$, suggesting that this batch suffers no compromise in ductility or tensile strength.

4.3.3. Ductility of Soft Wire. As expected, the soft wire batches of Figure 11 had very high ductility. For the standard $2.5 \mathrm{~mm}$ soft, the average ductility was between 10 and $14 \%$. This was further exceeded by the $2.25 \mathrm{~mm}$ wire reaching $16.7 \%$.

Ductility emerges as an unreliable indicator of wire quality, since some known problematic batches nonetheless had adequate ductility.

4.4. Young's Modulus. Young's modulus tests were only able to be performed on a small range of batches due to material (small quantities of wire) and time constraints. Figure 12 reveals that no significant trends exist with wire stiffness (Young's modulus). All of the Young's moduli tested fall within 140-160 GPa. These values are low compared to the usual modulus of steel of about $200 \mathrm{GPa}$. The reason for this apparent discrepancy could be due to the clamping system and/or the strain measurement system on the MTS 810 tensile machine. Further validation of these results is necessary if they are to be relied on. The fact that no trends exist between acceptable and problematic wire batches indicates that stiffness is not likely to be an important discriminator of wire quality.

4.5. Flexural Modulus. The flexural modulus property measures the ratio of stress to strain in bending. From a failure perspective, this property describes the stress that a member will experience for a given deflection. It is similar to the elastic 


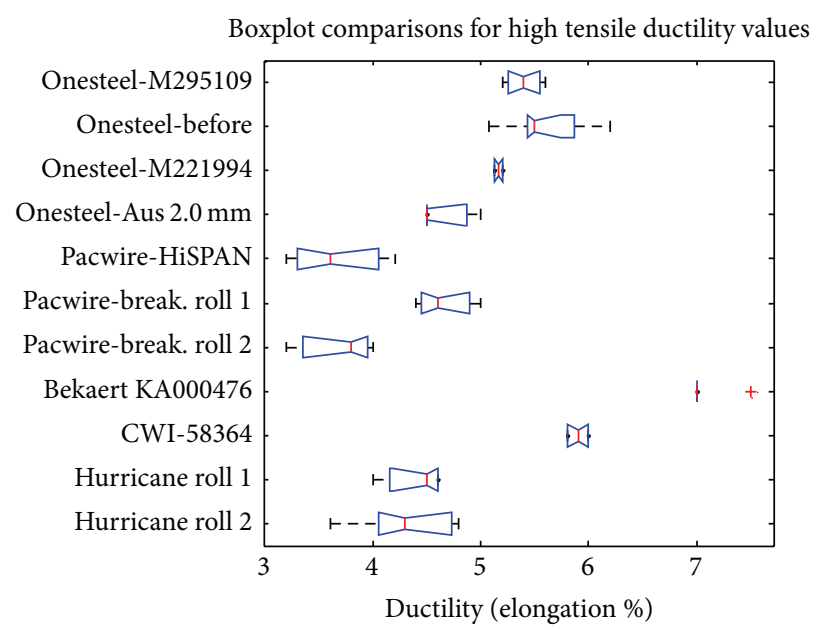

FIGURE 8: Boxplot comparison of ductility values for the various high tensile batches.

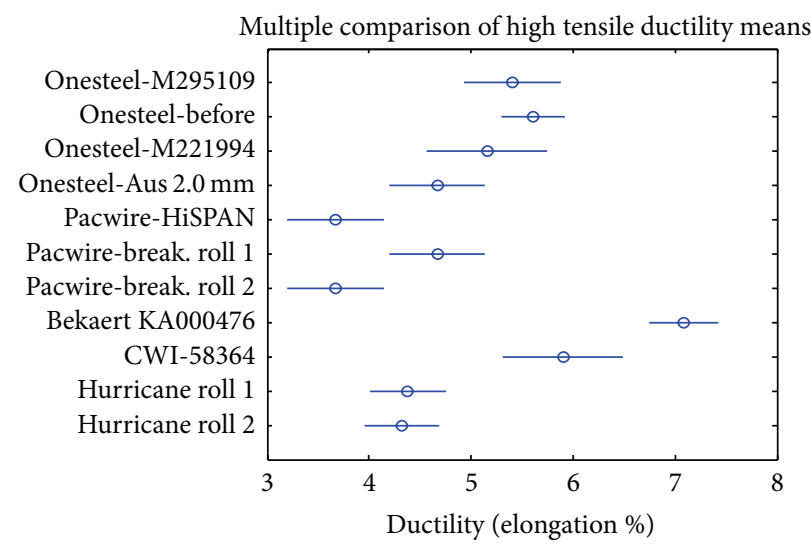

Figure 9: Multiple comparison of ductility means for high tensile wire batches.

modulus (or Young's modulus that measures the ratio of stress to strain in tension). However an important difference is that the flexural modulus includes the beam geometry and so is not so much a material property as a material-and-beam-size variable.

4.5.1. Method for Three-Point-Bending Test. To better simulate the conditions under which line wires are subjected in the knotting process, a three-point bending test was set up to determine the flexural modulus, $E_{f}$, of a wire sample in bending. The objective was to replicate the process whereby the line wire is bent around the vertical stay wires in an extreme loading situation. For a circular section the flexural modulus is given by the formula

$$
E_{f}=\frac{4 L^{3} m}{3 \pi d^{4}}
$$

where $L$ is the span between supports ( $\mathrm{mm}), m$ is the slope of the tangent to the initial straight-line portion of the loaddeflection curve $(\mathrm{N} / \mathrm{mm}), d$ is the diameter of wire $(\mathrm{mm})$, and $E_{f}$ is the flexural modulus in bending $(\mathrm{MPa})$.

The three-point bend test was based on the standard: ASTM D790: test methods for flexural properties of plastics [22]. Since plastics were not being tested, only relevant sections were implemented. The tests were carried out using a custom built bending rig fitted to a MTS 810 Material Testing System. The base supports were fully adjustable, allowing the bending span to be altered. Figures 13 and 14 show the bending rig layout and dimensions.

For this investigation, a support span of $26 \mathrm{~mm}$ was used. This was chosen based on measuring the crimp dimensions of fabricated stiff-stay fences. A thin metal strip was used to apply the midspan force. Wire samples were cut to a length of $50 \mathrm{~mm}$ before being tested. Any knotted samples first had their knots carefully removed and were loaded "knot down" to try and exploit the presence of the notch. The MTS 810 Material Testing System was configured into compression mode, with a cross-head displacement rate of $-2 \mathrm{~mm} / \mathrm{minute}$. As a minimum, four wire samples from any one batch were tested. Plotting the load-deflection results allowed the flexural modulus values to be calculated. ANOVA (analysis of variance) statistical analysis techniques were then used to determine if "statistically significant" results existed.

4.5.2. Results for Flexural Modulus. The flexural modulus results from all tests are shown in Figure 15. A Boxplot showing a summary of the statistical results is shown in Figure 16. 
TABLE 3: Statistical results for ANOVA: single factor test.

(a) Summary

\begin{tabular}{lcccc}
\hline Groups & Count & Sum & Average $E_{f}$ & Variance \\
\hline Pacwire-HiSPAN & 4 & 143.254 & 35.814 & 1.677 \\
Pacwire-breaking 1 & 4 & 130.653 & 32.663 & 45.283 \\
Pacwire-breaking 2 & 4 & 132.910 & 33.227 & 20.576 \\
Onesteel B\# 295109 & 5 & 188.727 & 37.745 & 10.868 \\
Bekaert B\# KA00476/0101 & 4 & 140.213 & 35.053 & 7.232 \\
Bekaert B\# KA004770101 & 4 & 106.384 & 26.596 & 4.369 \\
Deacero B\# 090130-009 \& 014 & 4 & 92.908 & 23.227 & 9.081 \\
\hline
\end{tabular}

Significance level, $\alpha=5 \%$ (0.05).

(b) ANOVA

\begin{tabular}{lccccc}
\hline Source of variation & SS & df & MS & $F$ & $P$ value \\
\hline Between groups & 691.26 & 6 & 115.21 & 8.226 & $9.616 E-05$ \\
Within groups & 308.12 & 22 & 14.01 & & 2.549 \\
\hline Total & 999.38 & 28 & & & \\
\hline
\end{tabular}

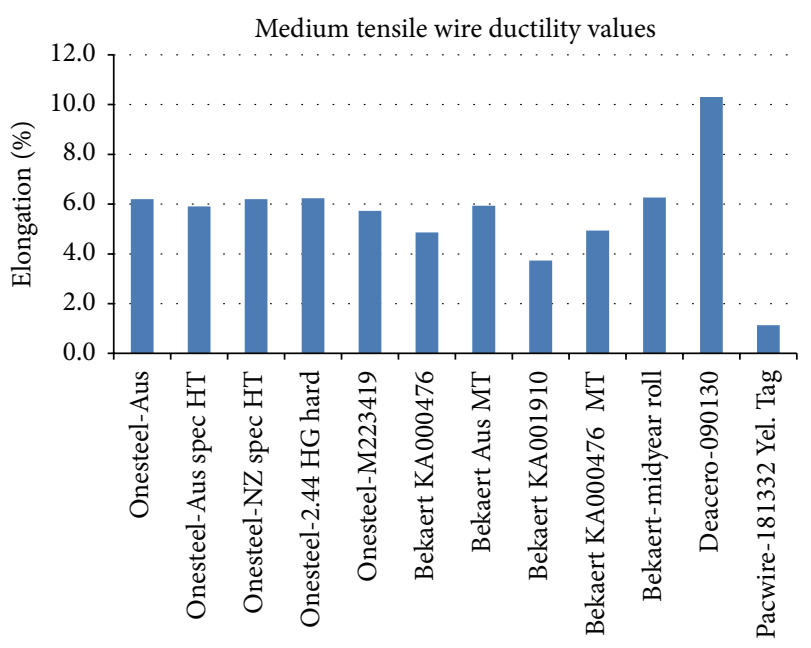

FIGURE 10: Average percent elongation results for a range of medium tensile wire batches.

The statistical ANOVA (analysis of variance) results are depicted in Table 3. To compare each of the wire batches against one another, a multiple comparisons investigation using confidence intervals was conducted. Two results can be said to be "statisitcally different" if there is no overlap of confidence intervals. Figure 17 depicts these results for a 95\% confidence level.

4.5.3. Discussion of Three-Point Bending. The three-point bending test results in Figure 15 show a high degree of variability, again confirmed by the boxplot in Figure 16. The source of this variability is unknown, since all known variables were maintained constant (such as span, cross-head displacement, etc.). One possible reason is that the MTS 810 Material Testing System was not sensitive enough for the testing of these wire samples in bending. The resulting loaddeflection graphs did contain load fluctuation, which made

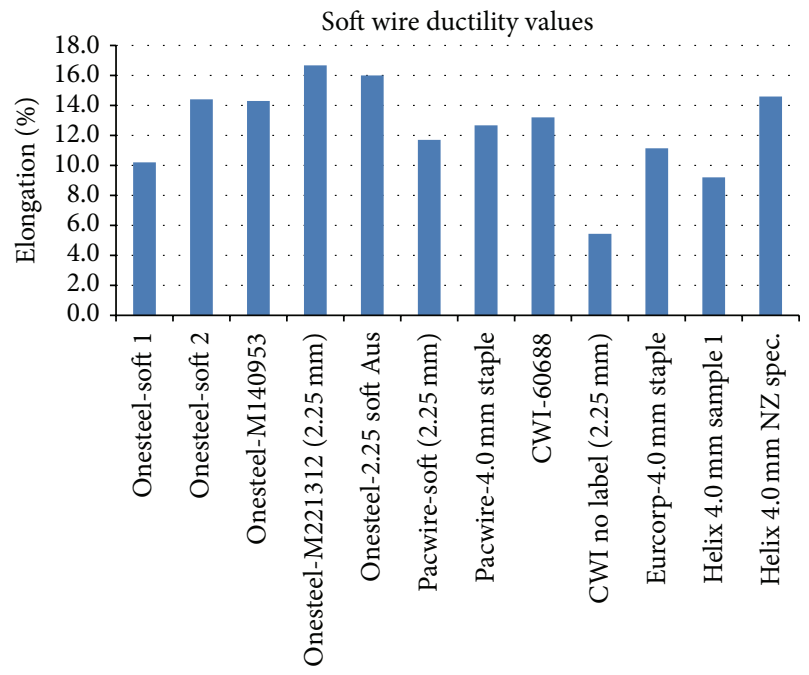

FIGURE 11: Average percent elongation results for a range of soft wire batches.

curve fitting to the initial portion of the graph more difficult. Since the flexural modulus is highly sensitive to variations in the initial slope, it is possible that the fluctuations are the source of the observed variability. The only way to test this would be to replicate the tests on a smaller, more sensitive compression testing setup.

The statistical ANOVA analysis gives a statistical test of whether the means of several groups are all equal (the null hypothesis). For this investigation, a significance level of $5 \%$ ( $\alpha=0.05$ ) was chosen. Table 3 shows that the $F$ value for the test (8.226) is greater than $F_{\text {crit }}(2.549)$. This gives a strong indication that the flexural moduli from the different wire brands/batches are not the same (i.e., null hypothesis can be rejected). An $F$ statistic as extreme as the observed $F$ would occur by chance one in 10,400 times if the means were truly equal. 


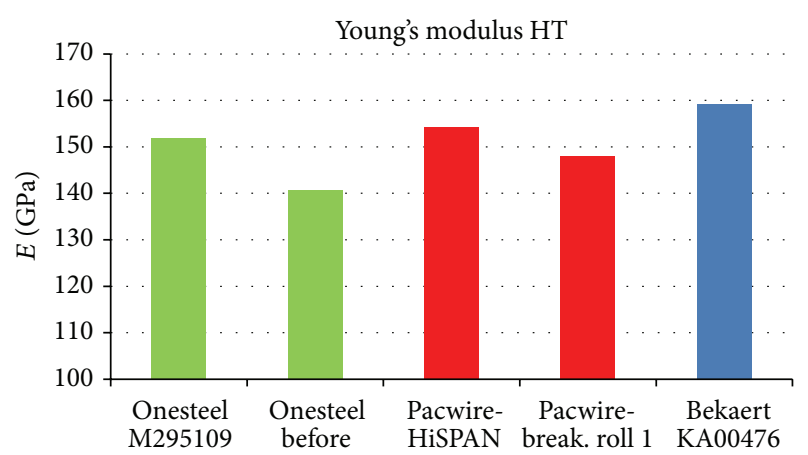

Figure 12: Average Young's modulus $(E)$ results for a range of high tensile wire.

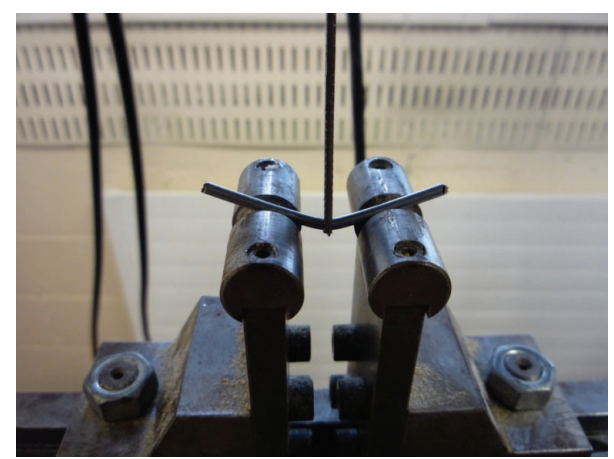

FIGURE 13: Three-point bending rig fitted to MTS 810 Testing System.

Since the ANOVA test cannot determine which particular pairs are "significantly different," a multiple comparison procedure was carried out, using the results from the ANOVA test. Figure 17 shows that there are seven significantly different mean pairs, the largest being between high tensile Onesteel and medium tensile Deacero wire batches.

Three-point bending is also an unreliable indicator of wire quality, since some known problematic batches nonetheless had high flexural modulus. Also, some known good batches had low modulus. The test thus has poor specificity and sensitivity.

4.5.4. Effect of Span on Flexural Modulus. During the threepoint bend testing, it was noted that many Pacific wire tests resulted in the wire sample fracturing when bent through an acute angle, while the majority of Onesteel, Bekaert, and Deacero wire did not. It was postulated that there might be a critical support spacing that caused all problematic Pacific wire to crack but leave all acceptable wire intact.

For the second part of this investigation, a pass/fail test was adopted, whereby Failis any visible cracking and Pass is no visible cracking. Wire samples from different batches were again cut to $50 \mathrm{~mm}$ lengths and loaded into the bending rig. The cross-head was manually controlled to bend the wire to the point of fracture, or as far as possible. This process was completed for varying support spans.

The bending span investigation results are as shown in Table 4. Bold font indicates fracture, italic font not fracture, and $\mathrm{m}$-dash not tested.

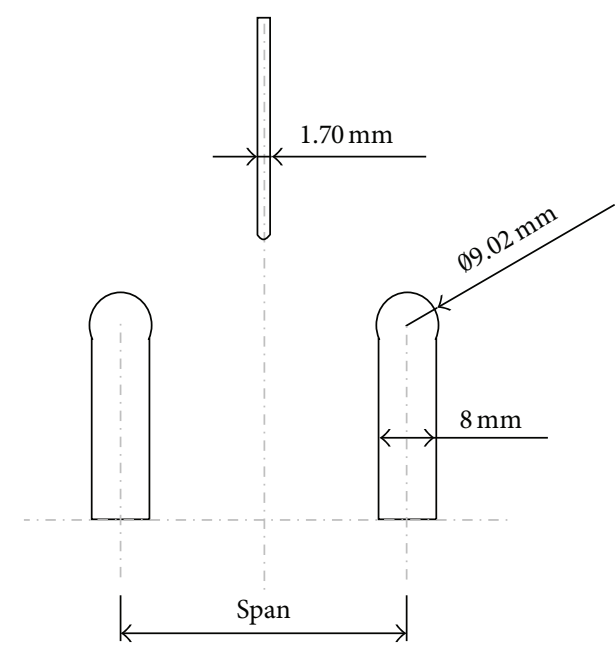

FIGURE 14: Rig dimensions for three-point-bending.

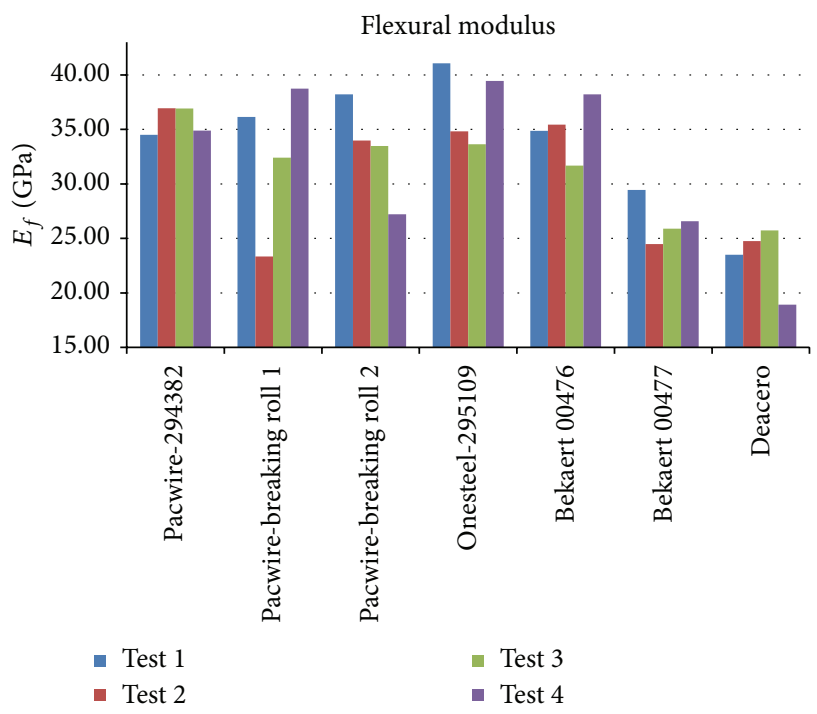

FIGURE 15: Results from all flexural modulus tests from a range of different batches/manufacturers.

The results show a number of interesting trends about the different wire brands/batches. They are as follows.

(i) All HiSPAN and breaking roll 2 Pacific wire batches fail for spans smaller than $22 \mathrm{~mm}$.

(ii) The majority of Onesteel samples fail for spans smaller than $21 \mathrm{~mm}$.

(iii) The majority of Bekaert samples remain intact even for the smallest span $(20.44 \mathrm{~mm})$.

(iv) Pacific wire breaking roll 1 begins failing at a span of $21 \mathrm{~mm}$.

These results do confirm the presence of critical support spacings. In general, the problematic Pacific wire batches tend to fracture at larger spans than the acceptable Onesteel and Bekaert wire batches. 


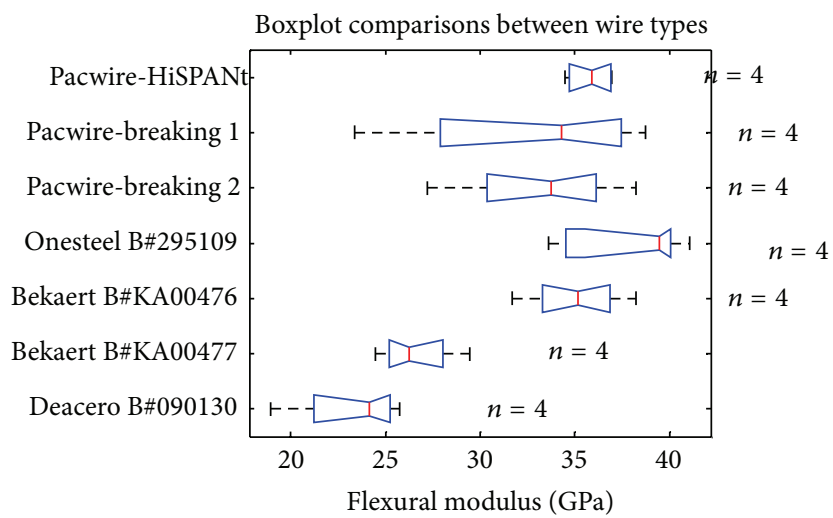

FIGURE 16: Boxplot comparisons between the various wire types.

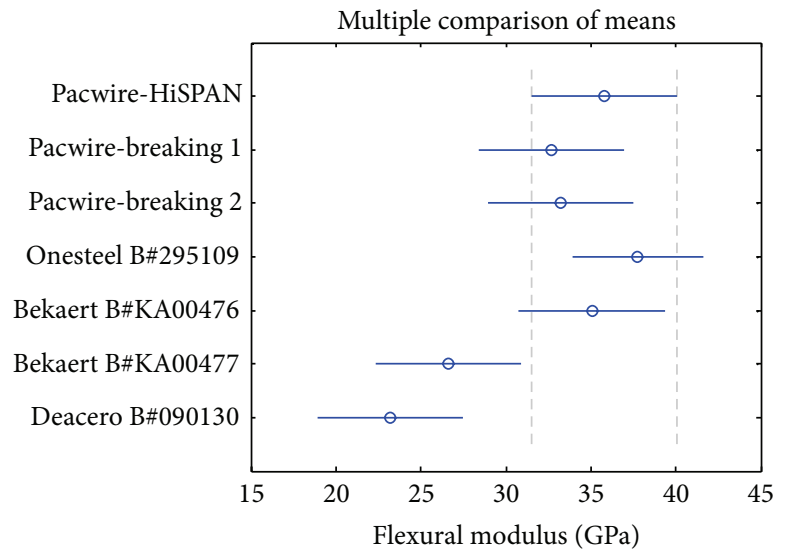

Figure 17: Multiple comparison of means between wire batches at 95\% confidence level.

This trend is violated by the Pacific wire breaking roll 1 specimens, which appear to have a better fracture tolerance than the acceptable Onesteel specimens do. The medium tensile Bekaert wire proved to be the best performer, only beginning to fracture at the smallest rig span of $20.44 \mathrm{~mm}$.

Span has some potential to be further developed into a test of wire quality, since the results were broadly consistent with the known quality of the wire (other than Pacwirebreaking roll 1). However it is not a quantitative test and the threshold is uncertain (it appears to be around $23 \mathrm{~mm}$ ). This is a potentially significant finding since the knotting of wire, for example, in the industrial fabrication of fences, or in-field tieoff, involves bending the wire to a tight radius of curvature. Often wire is turned back and wrapped around itself when knotted. Thus wire that passes other tests might fail in the knotting situation because of sensitivity to tight bending.

\section{Discussion}

5.1. Outcomes: What Has Been Achieved? A batch of wire samples, of different origins, has been subjected to a battery of tests for physical material-properties. These tests have included the standard tensile strength and elongation as presently used in the wire industry, as well as other tests that currently are not: Young's modulus, flexural modulus, and bending-radius (span) effects.

5.1.1. Comparison Across Brands. The results are generally based on multiple rather than single specimens, and this permitted various statistical tests to be performed. These show that there is indeed a statistically significant difference (at the 5\% significance level) between many of the brands for tensile strength, ductility, and flexural modulus.

5.1.2. Comparison to Known Wire Quality. Results were obtained for various material properties, for various wire brands. The quality of the wire was also known, and this permitted the sensitivity of the various tests to be determined.

Generally the multiple comparison analyses showed unreliable discrimination of the tests to known good and poor quality wire, in that the distribution of breaking wire overlapped the good wire. This problem was observed for tensile strength and to a lesser extent ductility and three-point bending. This is an issue because it means that the tests are not very powerful: they may let through wire that turns out to fail during production or fence erection (type II error).

5.1.3. Novel Contribution. The novel contribution of this work is first the publishing of multiple material properties 
TABLE 4: Bending span investigation results for range of wire batches.

\begin{tabular}{|c|c|c|c|c|c|}
\hline Sample & SPAN $1=26.06 \mathrm{~mm}$ & SPAN $2=24.01 \mathrm{~mm}$ & SPAN $3=21.96 \mathrm{~mm}$ & SPAN $4=21.00 \mathrm{~mm}$ & SPAN $5=20.44 \mathrm{~mm}(\max )$. \\
\hline \multicolumn{6}{|c|}{$\begin{array}{l}\text { Pacwire-breaking } \\
\text { roll } 1\end{array}$} \\
\hline Test 1 & No fracture & No fracture & No fracture & Fracture, no pop & Fracture, no pop \\
\hline Test 2 & No fracture & No fracture & No fracture & No fracture & No fracture \\
\hline Test 3 & No fracture & No fracture & No fracture & No fracture & Only galv. crack \\
\hline Test 4 & No fracture & No fracture & No fracture & Fracture, pop & Fracture, no pop \\
\hline Test 5 & No fracture & No fracture & No fracture & Fracture, pop & Fracture, pop \\
\hline Test 6 & - & - & - & No fracture & Fracture, pop \\
\hline \multicolumn{6}{|c|}{$\begin{array}{l}\text { Pacwire-breaking } \\
\text { roll } 2\end{array}$} \\
\hline Test 1 & Fracture & Fracture, pop & Fracture, pop & Fracture, pop & Fracture, pop \\
\hline Test 2 & Fracture, pop & No fracture & Fracture, no pop & Fracture, pop & Fracture, pop \\
\hline Test 3 & Fracture, pop & Fracture, pop & Fracture, pop & Fracture, pop & Fracture, pop \\
\hline Test 4 & No fracture & No fracture & Fracture, no pop & Fracture, pop & Fracture, pop \\
\hline Test 5 & No fracture & No fracture & Fracture, pop & Fracture, pop & Fracture, pop \\
\hline Test 6 & - & Fracture, pop & - & - & - \\
\hline \multicolumn{6}{|c|}{ Pacwire-HiSPAN } \\
\hline Test 1 & Fracture, pop & Fracture, pop & Fracture, pop & Fracture, pop & Fracture, pop \\
\hline Test 2 & No fracture & Fracture, pop & Fracture, pop & Fracture, pop & Fracture, pop \\
\hline Test 3 & Fracture, pop & Fracture, pop & Fracture, pop & Fracture, pop & Fracture, pop \\
\hline Test 4 & No fracture & Fracture, pop & Fracture, pop & Fracture, pop & Fracture, no pop \\
\hline Test 5 & Fracture, pop & Fracture, pop & Fracture, pop & Fracture, pop & Fracture, pop \\
\hline \multicolumn{6}{|c|}{ Onesteel line 7} \\
\hline Test 1 & No fracture & No fracture & Fracture, pop & Fracture, pop & No fracture \\
\hline Test 2 & No fracture & No fracture & No fracture & Fracture, loud pop & Fracture, pop \\
\hline Test 3 & No fracture & No fracture & Fracture, pop & Fracture, pop & Fracture, pop \\
\hline Test 4 & No fracture & No fracture & No fracture & Fracture, loud pop & Fracture, pop \\
\hline Test 5 & No fracture & Fracture, pop & No fracture & Fracture, pop & Fracture, pop \\
\hline \multicolumn{6}{|c|}{ Onesteel line 8} \\
\hline Test 1 & No fracture & No fracture & Fracture, pop & Fracture, pop & Fracture, pop \\
\hline Test 2 & No fracture & No fracture & Fracture, pop & Late Fracture, pop & Fracture, pop \\
\hline Test 3 & No fracture & No fracture & No fracture & No fracture & Fracture, pop \\
\hline Test 4 & No fracture & No fracture & No fracture & Fracture, pop & Fracture, pop \\
\hline Test 5 & No fracture & No fracture & No fracture & Fracture, pop & No fracture \\
\hline Test 6 & - & - & - & - & Fracture, pop \\
\hline \multicolumn{6}{|c|}{ Onesteel 295109} \\
\hline Test 1 & No fracture & No fracture & No fracture & Fracture, pop & Late fracture, pop \\
\hline Test 2 & No fracture & No fracture & No fracture & Late fracture, pop & Fracture, pop \\
\hline Test 3 & No fracture & No fracture & No fracture & Fracture, pop & Fracture, no pop \\
\hline Test 4 & No fracture & No fracture & No fracture & Fracture, pop & Fracture, pop \\
\hline Test 5 & No fracture & No fracture & No fracture & Fracture, pop & Fracture, loud pop \\
\hline \multicolumn{6}{|c|}{$\begin{array}{l}\text { Bekaert } \\
\text { KA00476-0101 }\end{array}$} \\
\hline Test 1 & No fracture & No fracture & No fracture & No fracture & No fracture \\
\hline Test 2 & No fracture & No fracture & No fracture & No fracture & No fracture \\
\hline Test 3 & No fracture & No fracture & No fracture & No fracture & No fracture \\
\hline Test 4 & No fracture & No fracture & No fracture & No fracture & Beginnings of crack (in galv.) \\
\hline Test 5 & No fracture & No fracture & No fracture & No fracture & Fracture, no pop \\
\hline
\end{tabular}


for fencing wire beyond only tensile strength and ductility, secondly the comparison of different batches of wire, and thirdly the categorisation of results by known wire quality in production.

5.2. Implications for Practitioners. The tensile testing results suggest that premature fence failures are unlikely to be caused solely by tensile properties. In general, the acceptable wire batches have displayed higher strengths, but the majority of the wire batches were above the minimum standard. However, neither the UTS nor ductility results have shown any clear distinction between acceptable and problematic wire quality. Ductility is the more powerful of the two, but this property alone cannot be relied on as a means of screening wire compatibility and quality.

For those practitioners who are specifying wire for input into a fence-knotting machine, the implication is that not all wire is created equal. The first issue is that not all wire on the market necessarily meets the existing standards for tensile strength and ductility. This is a production quality issue. The second issue is that the existing standards for tensile strength and ductility cannot be fully relied on to discriminate between good and poor wire quality. It is possible for wire to meet these tests and yet still fail in production [19]. Fence producers who are concerned about wire-quality would want to insist that their suppliers at least meet the existing tensile strength and ductility standards. Ductility appears to be the harder property to meet. There are also other tests, not yet codified into standards, that could be used [19].

Overall, it must be remembered that tensile specifications are fundamental to the wire classification system. Tensile properties therefore should still continue to be evaluated at all stages in the fabricated fence production sequence. Flexural modulus evaluations, on the other hand, appear to have no significant production implications.

5.3. Limitations. The work done here was based on samples of wire and fence. Due to the nature of the wire production process, it is to be expected that there will be differences in wire batches over time. Therefore the data and conclusions apply only to the batches tested over that window of time and not necessarily to the brands as a whole.

Sample sizes were constrained due to the quantity of wire provided. Some batch samples only contained enough wire to permit a bare minimum of three tensile tests, while other samples were much larger.

Also, the power of the results is only as good as the correct assignment of acceptable and problematic quality to wire. For example, the Pacific steel breaking roll 1 batch, despite being labelled as problematic wire by the end user, performed very well in the tensile tests. Not only was its tensile strength comparable with the acceptable batches, but so too was its ductility. It must also be noted that this particular batch performed acceptably in other tests, for example, LTD, threepoint bending, and impact energy [19], which indicates that the issues with this batch may have had more to do with the user than the wire itself.
5.4. Implications for Further Research. The first implication is that existing metrics of tensile strength and ductility are unreliable discriminators of wire quality, at least according to the supplied samples. One avenue for further research could be to repeat the tests with a bigger sample of known good and bad wire. This would be useful to quantify the statistical power of the various tests. However the results from the existing work already show that existing tests have poor power, and doing more work to know this with greater certainty might not be the most useful way to move the field forward. It would add greater value to have fundamentally better understanding of the failure mechanism for wire during fence-production and better tests of incoming wire quality. Nonetheless the point is that future work needs to ensure a ready supply of known good and bad material.

In general is has been observed that there is some inverse correlation between tensile strength and ductility. Medium and soft tensile grades have lower UTS values but benefit from having increased ductility. There does appear to be exception to this rule, with some of the high tensile Onesteel batches having excellent UTS values combined with respectable ductility. It is this combination of properties which is most suited to the fabrication of high quality fences. Therefore, some form of test could be developed that can reliably identify these wire batches.

As discussed above, ductility gives the more powerful measure of wire quality. It therefore may be that ductility can be combined into another test, which enhances the discriminating power. One method may include dropping the temperature below zero, thus inducing more brittle behaviour from the poorer quality wire batches (i.e., ductile-to-brittle transition phenomenon exhibited in metals). Furthermore there may be more suitable temperature dependant tests available such as an impact energy experiment, and indeed other work conducted by the research group addresses this and low-temperature effects.

\section{Conclusions}

The objective of this investigation was to investigate the material properties of various bathes of wire. The results showed that the majority of the high tensile wire samples met the NZS 3471:1974 minimum tensile requirements. Only the Pacific Steel HiSPAN and CWI batches failed to meet the UTS standard. In terms of ductility, again the Pacific steel HiSPAN and breaking roll 2 batches failed to meet the minimum 4\% ductility. Young's Modulus results revealed no major differences. Three-point bending and span effects showed significant differences between batches.

However, the bigger question is the power of the tests to discriminate between good and poor wire quality. While there was significant variability between wire batches, this did not correlate reliably with the known wire quality. Therefore it is concluded that existing test methods, including tensile strength and ductility, are somewhat incomplete, perhaps even unreliable, as measures of wire quality. 


\section{Conflict of Interests}

The authors declare that there is no conflict of interests regarding the publication of this paper.

\section{Authors' Contribution}

All authors contributed to the work and writing of the paper.

\section{References}

[1] Anon, "Ring-lock wire-fence making machine," Engineering, vol. 133, no. 3452, p. 309, 1932.

[2] S. S. DeForest, "Development of fence erecting machine," Agricultural Engineering, vol. 42, no. 2, pp. 66-67, 1961.

[3] C. Hamann, "Wire crimping," Draht, vol. 12, no. 4, pp. 155-160, 1961.

[4] C. E. Hann, D. Aitchison, D. Kirk, and E. Brouwers, "Modelling and system identification of a stiff stay wire fence machine," Proceedings of the Institution of Mechanical Engineers, Part B (Journal of Engineering Manufacture), vol. 224, no. B7, pp. 10691083, 2010.

[5] Australian Standards, AS 1650-1989 Hot-Dipped Galvanized Coatings on Ferrous Articles, Australian Standards, Sydney, Australia, 1650.

[6] SNZ, "NZS 3471:1974A1-Specification for Galvanised Steel fencing wire," NZS 3471, Standards New Zealand, Wellington, New Zealand, 1974.

[7] BSI, Specification for Fences, Part 10, British Standards Institution-British Standard, 1963.

[8] ASTM, Annual Book of ASTM Standards, 1981, Part 3: Steel Plate, Sheet, Strip And Wire; Metallic Coated Products; Fences, Annual Book of ASTM Standards, Part 3, ASTM, 1981.

[9] Australian Standards, AS 1650-1989 Hot-Dipped Galvanized Coatings on Ferrous Articles, Australian Standards, 1650.

[10] Anon, "Atmospheric corrosion tests on wire and wire products begun by A.S.T.M," Steel, vol. 99, no. 25, p. 5154, 1936.

[11] W. H. Bleecker, "Choice of fencing material affected by atmospheric conditions," Oil and Gas Journal, vol. 36, no. 51, p. 48, 1938.

[12] C. C. Crane, "New electro-galvanizing process for round wire," Steel, vol. 100, no. 19, pp. 71-72, 1937.

[13] B. A. Jennings, "Fence exposure tests," Agricultural Engineering, vol. 25, no. 4, pp. 140-141, 1944.

[14] F. M. Reinhart, "Twenty-year atmospheric corrosion investigation of zinc-coated and uncoated wire and wire products," Special Technical Publications 290, American Society for Testing and Materials, 1961.

[15] J. L. Schueler, "New process for making zinc coated farm fencing more durable," Agricultural Engineering, vol. 14, no. 12, pp. 339340, 1933.

[16] O. W. Storey, The Corrosion of Fence Wire, American Electrochemical Society, 1917.

[17] R. Nuenninghoff and K. Sczepanski, "Galfan-an improved corrosion protection for steel wire. Part 2: applications of aluminium-zinc coated steel wires and tests with coated products," Wire, vol. 37, no. 4, pp. 321-324, 1987.

[18] R. Fabien, M. Robertson, and A. V. Nguyen, "Study on parameters influencing the corrosion of metallic coatings on wire exposed to marine environments," Wire Journal International, vol. 40, no. 7, pp. 94-98, 2007.
[19] D. Pons, G. Bayley, R. Laurenson, M. Hunt, C. Tyree, and D. Aitchison, "Wire fencing (part 1): determinants of wire quality," The Open Industrial \& Manufacturing Engineering Journal, vol. 5, no. 5, pp. 19-27, 2012.

[20] ASTM, ASTM A370-05 Standard Test Methods and Definitions for Mechanical Testing of Steel Products, A370, American Society for Testing and Materials (ASTM), 2005.

[21] British Standards Institute (BSI), "BS EN 10223-5:1998-steel wire and wire products for fences. Steel wire woven hinged joint and knotted mesh fencing," Tech. Rep. BS EN 10223, British Standards Institute, London, UK, 1998.

[22] ASTM, STM D790-07e1 Standard Test Methods for Flexural Properties of Unreinforced and Reinforced Plastics and Electrical Insulating Materials, American Society for Testing and Materials (ASTM), 2007. 

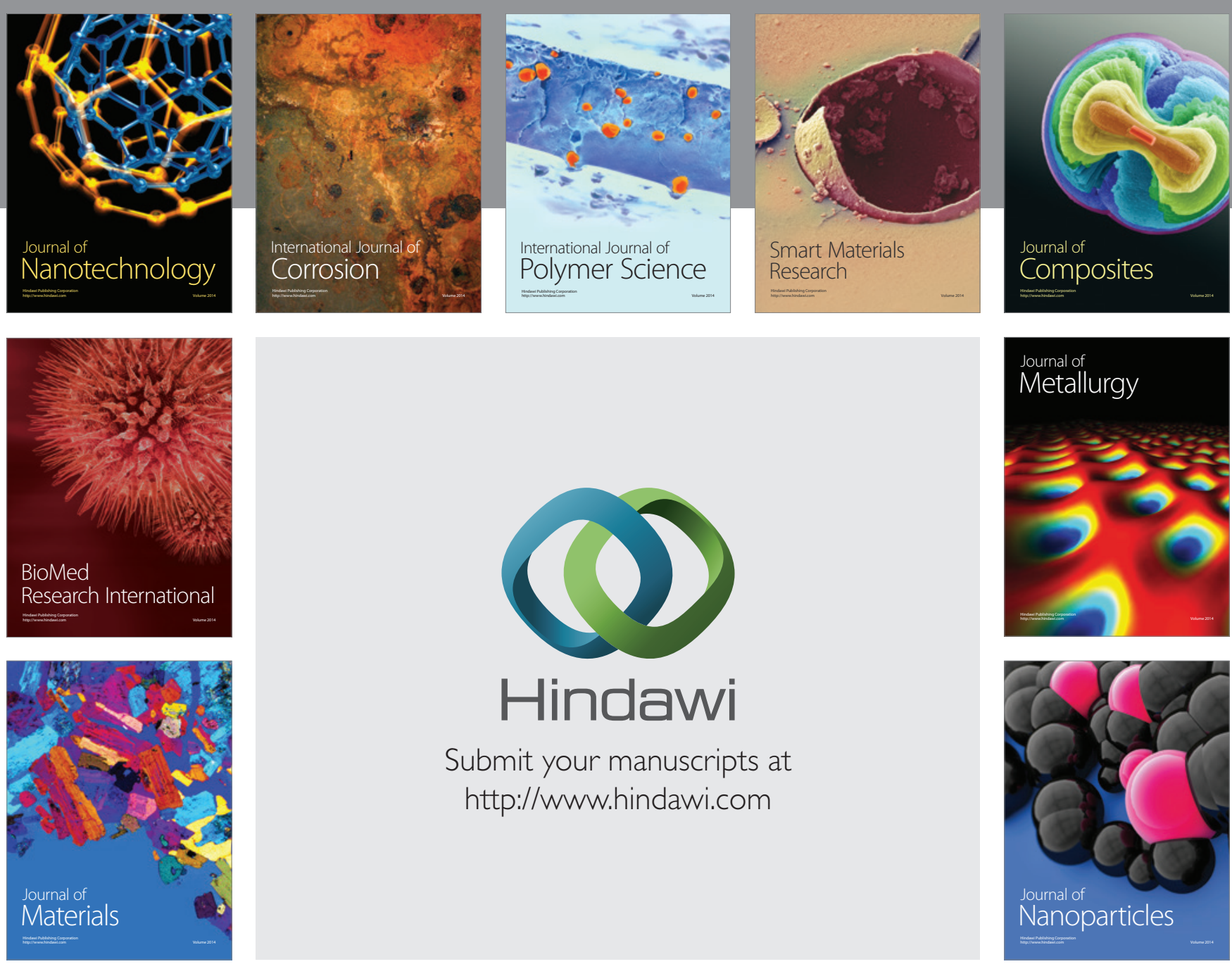

Submit your manuscripts at http://www.hindawi.com
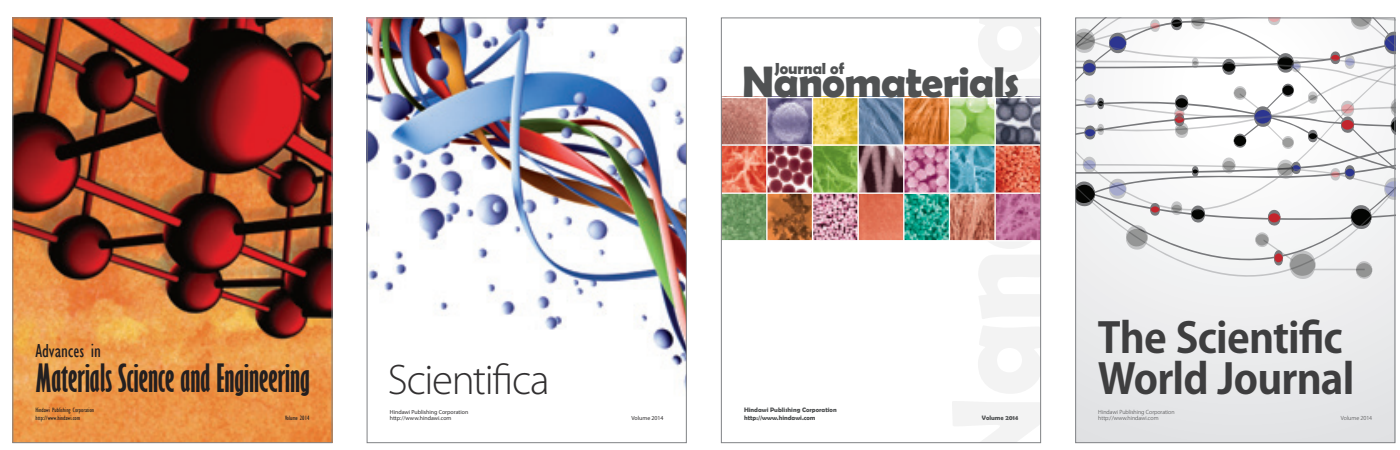

\section{The Scientific World Journal}
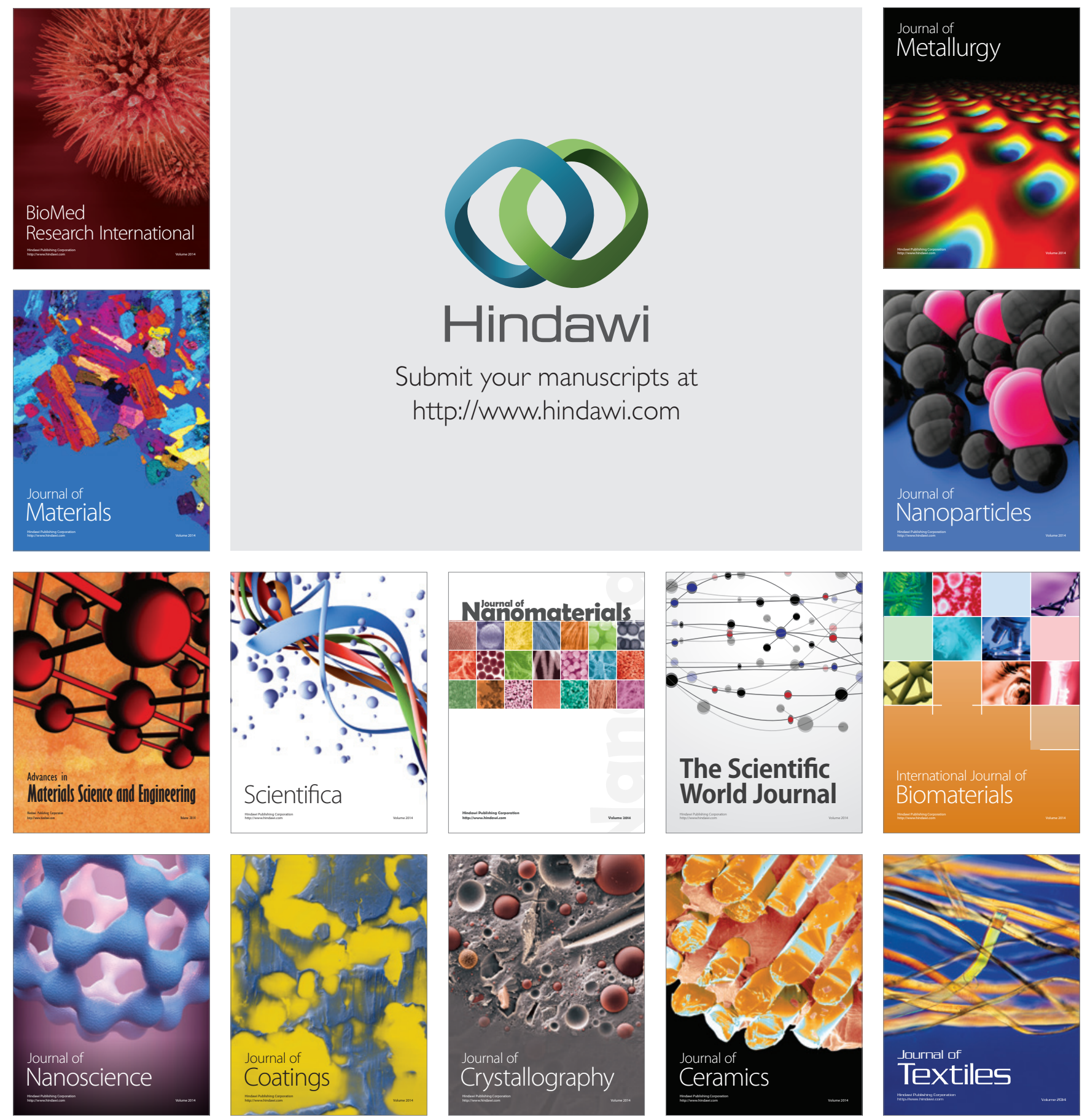